keen examination of theory, a critical disposition, and above all it is interesting and engaging.

Femi Oyebode Professor of Psychiatry, University of Birmingham, National Centre for Mental Health, 25 Vincent Drive, Edgbaston, Birmingham B15 2FG, UK. Email: Femi.Oyebode@bsmhft.nhs.uk

doi: 10.1192/bjp.bp.112.114652

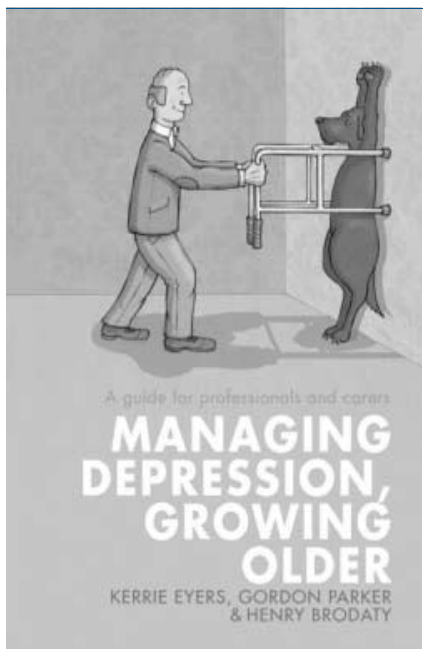

Managing Depression, Growing Older: A Guide for Professionals and Carers

By Kerrie Eyers, Gordon Parker \& Henry Brodaty Allen \& Unwin. 2012. AUD $\$ 27.99(\mathrm{pb}), 300 \mathrm{pp}$ ISBN: 9781742378800

Old age psychiatry has experienced a change in its culture. With the ageing population predicted to rise exponentially in the coming years, we constantly strive to be ready for the dementia 'time bomb' and to find adequate resources for those already in the midst of the illness. To a clinician, it can at times feel as if functional illness in older age is being forgotten. This guide, edited and produced by the Australian Black Dog Institute, is a selfpurported 'timely' book which aims to raise the awareness of depression in older age. It promises to challenge the common misperception that, by necessity, ageing brings with it the 'black dog of depression'.

The book is an easy-to-digest mixture of evidence-based science and personal narrative. The range of subjects explored within is vast. The authors move deftly and coherently between topics, varying from how to diagnose depression to how to age well. A humorous quotation begins each chapter and serves to promote positivity. After establishing the topic in easy-tounderstand science, the focus turns to personal reflections of depression. Each chapter concludes with a 'noteworthy' section, providing the reader with a few practical points about coping with and managing depression in later life.

The sheer range of topics means that some are explored superficially, making this a book to recommend to junior colleagues looking for an introduction to the subject, carers or patients (although perhaps those who are not acutely unwell) rather than senior psychiatrists wanting to expand their knowledge base. That said, the personal reflections which account for the majority of the text are the great strength of this book. Collected from essays submitted to the Black Dog Institute and contributions invited from eminent professionals, each one of these wonderfully written pieces conveys something different about what it means to have been 'bitten by the black dog. Although often saddening to read, they easily connect the reader to the experience of depression in older age and instil a sense of something valuable and inspirational about having lived with that experience. For this, the book is an important addition to our resources and a reminder that we should expect good mental health with ageing. The accounts are written articulately, purposefully and emotionally and offer perspectives from patients, relatives, carers and professionals, making this an insightful and accessible book for all.

Emma K. Parry ST5 in Old Age Psychiatry, and Honorary Clinical Lecturer, University of Birmingham, UK. Email: emma.parry@hacw.nhs.uk

doi: 10.1192/bjp.bp.112.115634

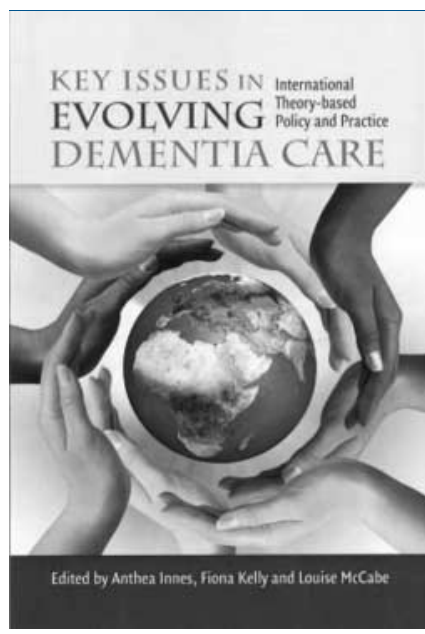

\section{Key Issues in Evolving Dementia Care: International Theory-based Policy and Practice}

Edited by Anthea Innes, Fiona Kelly \& Louise McCabe Jessica Kingsley Publishers. 2012. £24.99 (pb). 256 pp. ISBN: 9781849052429

This edited volume takes a welcome approach to review current issues in dementia care, providing a broad-based view of the conceptualisation of dementia, policy and practice. The editors devote space to developments in national contexts as far apart as the Deep South of the USA, Malta and India, as well as Europe, giving readers a chance to compare notes and pick up tips. They have taken care to attend to the voices of people with dementia and to grassroots initiatives as well as overarching perspectives, bringing the subject matter closer to home for the average practitioner than many more erudite volumes. In today's language this could be described as a volume concerned with knowledge transfer and impact.

The collection of chapters grew from a 21st birthday celebration for the ground-breaking Dementia Services Development Centre at the University of Stirling. As such, the material seems to have an element of serendipity rather than a priori intention. This leads to some odd bedfellows: a useful scholarly chapter reviewing evidence for population-level influences on dementia (chapter 3 ), for example, is close neighbours with another that reviews development and implementation of dementia policies in France (chapter 6) from a much more narrative and personal perspective.

Reading about implementation of policy and theory in practice leads to the exposure of a number of thought-provoking 'gaps'. These include the 'dementia gap', a term given by the Alzheimer's Society to the difference between the number of people estimated to have dementia and the number who have received a diagnosis (see chapter 4 ), and policy practice gaps revealed in the chapters on strategy, which include a fluent and honest account of the situation in England (chapter 5). We also 